\title{
A White Light Reflectance Spectroscopy Label-Free Biosensor for the Determination of Fungicide Carbendazim ${ }^{+}$
}

\author{
Georgios Koukouvinos ${ }^{1, \ddagger}$, Chrysoula-Evangelia Karachaliou ${ }^{2, *, \neq}$, Sotirios Kakabakos ${ }^{1}$ and \\ Evangelia Livaniou $2, *$ \\ 1 Immunoassay/Immunosensors Lab, INRASTES, NCSR “Demokritos”, Athens, Greece; \\ email1@gmail.com (G.K.); email1@gmail.com (S.K.) \\ 2 Immunopeptide Chemistry Lab, INRASTES, NCSR “Demokritos”, Athens, Greece; \\ * Correspondence: xrisak15@hotmail.com (C.-E.K.); livanlts@rrp.demokritos.gr (E.L.); Postal Address: \\ National Centre for Scientific Research “Demokritos” P.O. BOX 60037, \\ 15310 Agia Paraskevi, Greece \\ † Presented at the 1st International Electronic Conference on Biosensors, 2-17 November 2020; Available \\ online: https://iecb2020.sciforum.net/. \\ $\ddagger$ Equal Contribution.
}

Received: date; Accepted: date; Published: date

\begin{abstract}
Carbendazim is a broad-spectrum benzimidazole-type fungicide effective against fungi that compromise the safety/quality of food products. Despite its potential usefulness, carbendazim constitutes a major environmental pollutant, being hazardous for humans and animals; therefore, reliable determination of carbendazim levels in water, soil and food samples remains a highly desirable analytical goal. Herein, an optical (white light reflectance spectroscopy, WLRS) label-free biosensor for fast and sensitive determination of carbendazim is presented. The transducer is a $\mathrm{SiO}_{2} / \mathrm{Si}$ chip, on which a suitable benzimidazole-conjugate has been immobilized; determination is based on the competitive immunoassay format: a mixture of an in-house developed anticarbendazim antibody with the calibrators/samples is pumped over the chip, followed by biotinylated secondary antibody and unlabeled streptavidin. The WLRS platform allows for realtime monitoring of biomolecular interactions carried out onto the $\mathrm{SiO}_{2} / \mathrm{Si}$ chip by transforming the shift in the reflected interference spectrum caused by the immunoreaction to effective biomolecular adlayer thickness. The sensor is capable of detecting carbendazim levels within 28 min (LoD: 20 $\mathrm{ng} / \mathrm{mL}$; intra- and inter-assay CVs: $\leq 6.9 \%$ and $\leq 9.4 \%$, respectively). Excellent analytical characteristics and short analysis time combined with its small size render the proposed WLRS biosensor ideal for future point-of-need determination of carbendazim in food and environmental samples.
\end{abstract}

Keywords: white light reflectance spectroscopy; label-free immunosensor; pesticides; carbendazim

\section{Introduction}

Fungal pathogens cause significant damages to the food crops every year resulting in poor yield, deficient food quality and huge economic loss; therefore, the use of fungicides gains ground to circumvent these problems [1]. Carbendazim (methyl 1H-benzimidazol-2-ylcarbamate) is a low molecular weight (191.19), systemic, broad-spectrum, benzimidazole-type fungicide (Scheme 1), used worldwide as pre- and post-harvest treatment to control fungi that compromise the quality of various vegetables, fruits, cereals and seeds [2,3]. Despite the unquestionable benefits provided to crop yield, carbendazim is a major pollutant, which induces acute and late effects on humans and livestock. In Proceedings 2020, 4, $\mathrm{x}$; doi: FOR PEER REVIEW 
this context, carbendazim has been documented to induce infertility, embryotoxicity, teratogenicity, hepatocellular dysfunction, endocrine-disrupting effects, disruption of haematological functions and mutagenicity [2]. Additionally, the World Health Organization (WHO) has classified carbendazim in group 2B, as possible human carcinogen [4]. Due to its afore-mentioned severe toxicities and its persistence in food and the environment, carbendazim has been officially banned in most of European Union, USA and Australia. However, some countries, such as UK, Portugal, India, China and Brazil are still permitting the production and use of carbendazim in various formulations [5], raising a growing concern for the health and safety of humans and animals.
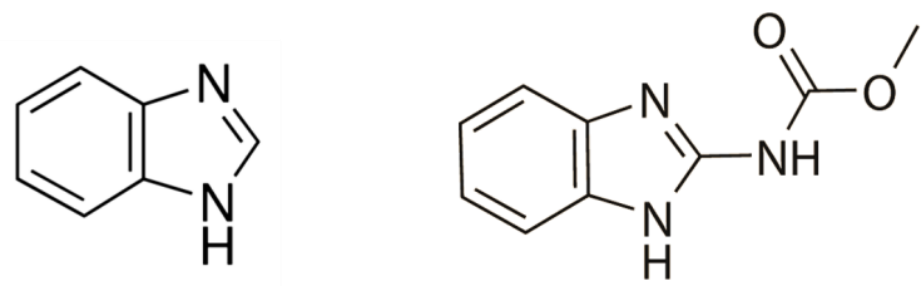

Scheme 1. Chemical structures of benzimidazole core-moiety (left) and carbendazim (right).

Analytical methodologies that ensure fast and accurate determination of carbendazim are a prerequisite to guarantee protection of public health. Determination of carbendazim is routinely performed by instrumental analysis techniques, mainly high performance liquid chromatography (HPLC) coupled to mass spectroscopy (MS) or ultraviolet spectroscopy (UV) [6,7]. Alternatively, immunochemical techniques have been developed and used, offering low cost- and short timeanalyses, simple assay protocols and minimum sample pre-treatment, as well as high-throughput sample capacity. In this context, classic enzyme-linked immunosorbent assays (ELISA) and immunochromatographic strips have appeared in the literature [8,9], while the modern technologies of biosensors have lately attracted much attention due to their simplicity, rapidity, portability and potential of point-of-need application [10].

In this work, a label-free sensor based on White Light Reflectance Spectroscopy (WLRS) is presented for the fast and sensitive determination of carbandazim. The transducer employed is a $\mathrm{Si}$ chip with a $1 \mu \mathrm{m}$ thick thermal $\mathrm{SiO}_{2}$ on top, which is transformed to a biosensing element through immobilization of a suitable benzimidazole conjugate on the $\mathrm{SiO}_{2}$ surface. Carbendazim determination is based on the competitive immunoassay format, i.e., a mixture of the antibody with the calibrators or the samples is pumped over the chip surface followed by reaction with biotinylated secondary antibody/streptavidin for signal enhancement. The WLRS biosensing platform allows for the label-free, real-time monitoring of biomolecular interactions carried out onto the $\mathrm{SiO}_{2} / \mathrm{Si}$ chip by transforming the shift in the reflected interference spectrum caused by the immunoreaction to effective biomolecular adlayer thickness. The developed sensor is capable of real-time detection of carbendazim with LoD: $20 \mathrm{ng} / \mathrm{mL}$, within $28 \mathrm{~min}$ total analysis time.

\section{Materials and Methods}

\subsection{Reagents and Chemicals}

Four-inch Si wafers $(<100>)$, purchased from Si-Mat Germany (Kaufering, Germany), were cleaned with acetone and isopropanol and then a $1-\mu \mathrm{m}$ thick $\mathrm{SiO}_{2}$ layer was grown on them by thermal oxidation at $1100{ }^{\circ} \mathrm{C}$ in the clean room facility of the Institute of Nanoscience and Nanotechnology of NCSR "Demokritos". Rabbit polyclonal antibody against carbendazim (anticarbendazim antibody) and benzimidazole-conjugate were in-house developed, as previously described [11]. Carbendazim pestanal ${ }^{\circledR}$, biotinylated goat anti-rabbit IgG (biotinylated secondary antibody), streptavidin, highly pure ethanol, acetone, isopropanol and (3-aminopropyl) triethoxysilane (APTES) were obtained from Sigma-Aldrich (St. Louis, MO, USA). Bovine serum albumin (BSA) was purchased from Acros Organics (Geel, Belgium). IgG Elution buffer was from 
Thermo FisherScientific Inc. (Waltham, MA, USA). Salts and other chemicals were purchased from Sigma or Merck. The water used throughout the study was distilled.

\subsection{WLRS Instrumentation}

In the present work, an optical label-free sensor is presented based on White Light Reflectance spectroscopy (WLRS). WLRS methodology involves a visible/near infra-red light source (ThetaMetrisis SA) and a miniaturized USB controlled spectrometer (Ocean Optics Inc.), operating in the corresponding spectral range. The white light emitted from the light source is guided to a reflection probe (ThetaMetrisis SA), which delivers the incident light to the biomodified surface and collects the reflected light directing it to the spectrometer. The surface consists of a transparent $\mathrm{SiO}_{2}$ film over a Si reflecting substrate covered by a custom designed microfluidic cell (Jobst Technologies $\mathrm{GmbH}$ ) providing the fluidic connections to the solutions. The particular sensor allows the label-free and real-time monitoring of the biomolecular reactions by recording the shift in the interference spectrum caused by the increase of the effective thickness of the biomolecular layer on the sensing surface during immunoreaction.

\subsection{Preparation of Calibrators}

Carbendazim stock solution of $5 \mathrm{mg} / \mathrm{mL}$ was prepared in pure ethanol, aliquoted and stored at $-30{ }^{\circ} \mathrm{C}$. Calibrators with concentrations ranging from $20 \mu \mathrm{g} / \mathrm{mL}$ to $20 \mathrm{ng} / \mathrm{mL}$ were prepared in $10 \mathrm{mM}$ phosphate buffer, $\mathrm{pH} 7.4$, containing $0.9 \% \mathrm{NaCl}, 0.4 \%(w / v)$ BSA and $5 \%(v / v)$ ethanol (assay buffer).

\subsection{Biochip Preparation and Assay Performance}

Chips were first cleaned and hydrophilized by $\mathrm{O}_{2}$ plasma treatment (10 mTorr) for $30 \mathrm{~s}$ in a Reactive Ion Etcher. Then, they were immersed for $20 \mathrm{~min}$ in a $2 \%(v / v)$ aqueous APTES solution, gently washed with distilled water, dried under a nitrogen $\left(\mathrm{N}_{2}\right)$ stream and cured by heating at 120 ${ }^{\circ} \mathrm{C}$ for $20 \mathrm{~min}$. Chips were kept at room temperature (RT) in a desiccator for at least $48 \mathrm{~h}$ prior to use. For the chip biofunctionalization, a suitable benzimidazole conjugate was deposited on the chips and incubated overnight at RT. The following day, chips were rinsed with phosphate buffer (washing buffer), blocked with immersion for $3 \mathrm{~h}$ in $2 \%(w / v)$ BSA solution in washing buffer, rinsed with washing buffer and distilled water, dried with $\mathrm{N}_{2}$ and used for the assay.

Prior to assay, each biofunctionalized chip was assembled with the fluidic module, placed on the docking station and equilibrated with assay buffer to acquire a stable baseline. For the assay, 1:1 $v / v$ mixtures of calibrators $(0.02-20 \mu \mathrm{g} / \mathrm{mL}$ in assay buffer $)$ with the rabbit anti-carbendazim antibody $(2 \mu \mathrm{g} / \mathrm{mL}$ in assay buffer) were passed over the chip for $18 \mathrm{~min}(50 \mu \mathrm{L} / \mathrm{min}$ flow rate), followed by a biotinylated anti-rabbit IgG antibody (7 $\mathrm{min})$, and streptavidin ( $3 \mathrm{~min}$ ). Finally, the biochip was regenerated by passing IgG elution buffer, followed by re-equilibration with assay buffer. The calibration curve was created by plotting the effective thickness of the built-up biomolecular layer (signal) corresponding to different calibrators, Sx (expressed as percentage of the zero-calibrator signal -maximum signal; $S_{0}$ ), for each one analyte against the respective analyte concentration in the calibrator solution.

\section{Results and Discussion}

\subsection{Assay Optimization}

In order to achieve a fast and sensitive immunochemical detection of carbendazim, many assay parameters had to be optimized. Due to the competitive nature of the assay, the two most crucial parameters to optimize were the concentration of benzimidazole-conjugate used for coating and the concentration of anti-carbendazim antibody, since their combination determines the highest signal (zero calibrator signal) and the assay sensitivity. Hence, based on preliminary titration experiments using different concentrations of the benzimidazole-conjugate $(100-1000 \mu \mathrm{g} / \mathrm{mL})$ in combination with different concentrations of the anti-carbendazim antibody $(0.5-5 \mu \mathrm{g} / \mathrm{mL})$, the highest analytical signal 
was obtained when $500 \mu \mathrm{g} / \mathrm{mL}$ benzimidazole-conjugate was employed for surface functionalization in combination with $4 \mu \mathrm{g} / \mathrm{mL}$ concentration of anti-carbendazim antibody. Nevertheles, an antibody concentration of $2 \mu \mathrm{g} / \mathrm{mL}$ was adopted in the final protocol, since this resulted in an adequately high zero-calibrator signal along with signifinantly improved assay sensitivity. Another parameter to optimize was the duration of the whole assay, in order to achieve a fast and reliable analysis. As shown in Figure 1a, a total analysis time of $28 \mathrm{~min}$ was adopted, consisting of $18 \mathrm{~min}$ for the primary immunoreaction, $7 \mathrm{~min}$ for the secondary immunoreaction and $3 \mathrm{~min}$ for the biotinylated secondary antibody-streptavidin reaction.

\subsection{Assay Characteristics}

A typical calibration curve obtained from the WLRS biosensing system for carbendazim is shown in Figure $1 b$.

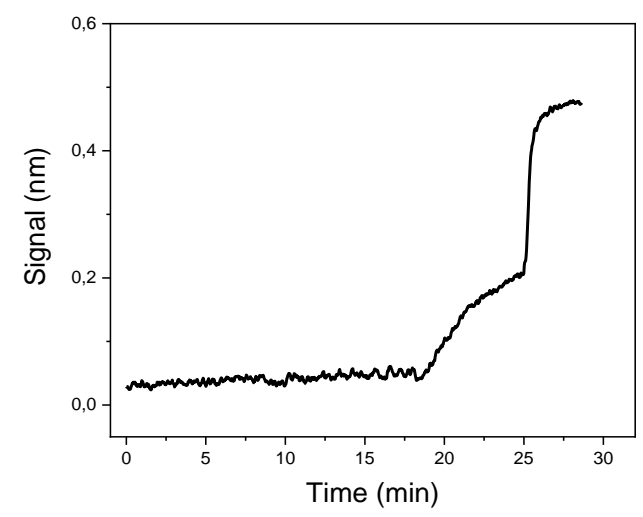

(a)

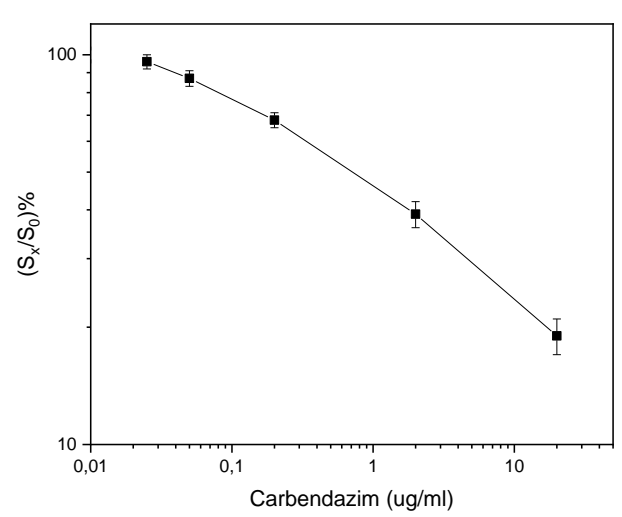

(b)

Figure 1. (a) Real-time response curve obtained from a biochip in the presence of zero calibrator, applying the optimized immunochemical protocol (b) Typical calibration curve obtained with carbendazim calibrators prepared in assay buffer. Each point represents the mean value of three independent runs \pm SD. $S_{0}=$ zero-calibrator signal; $S_{x}=$ calibrator signal.

The main analytical characteristics of the immunochemical detection of carbendazim using the WLRS sensor are summarized in Table 1. As shown, the analytical sensitivity of the proposed immunosensor was evaluated through calculation of the assay limit of detection (LoD) corresponding to mean value -3SD of 15 replicate measurements of zero calibrator, and was found to be $20 \mathrm{ng} / \mathrm{mL}$, whereas the dynamic range extended up to $20 \mu \mathrm{g} / \mathrm{mL}$. The reproducibility of the assay was assessed by running three control samples in triplicate within the same day, and also in seven different days, in order to calculate the intra- and inter-assay coefficients of variation $(\mathrm{CV})$, respectively. The intraassay $\mathrm{CV}$ values were $\leq 6.9 \%$, while the inter-assay $\mathrm{CVs} \leq 9.4 \%$.

Table 1. Immunochemical detection of carbendazim using the WLRS biosensing platform: Assay characteristics.

\begin{tabular}{cc}
\hline Assay Characteristic & Value \\
\hline LoD (ng/mL) & 20 \\
Dynamic range (ng/mL) & up to 20000 \\
Intra-assay CVs (\%) & $\leq 6.9$ \\
Inter-assay CVs (\%) & $\leq 9.4$ \\
Duration & $28 \mathrm{~min}$ \\
\hline
\end{tabular}

\section{Conclusions}

Herein, a WLRS-based biosensing platform for the label-free and real-time immunochemical determination of carbendazim was successfully developed. The proposed sensor allowed for the 
sensitive and fast quantification of carbendazim levels down to $20 \mathrm{ng} / \mathrm{mL}$ within less than $30 \mathrm{~min}$. The proposed biosensor is planned to be applied for the determination of carbendazim in samples of interest, e.g., citrus fruit juices. Taken it altogether, excellent analytical characteristics and short analysis time combined with small size of the analytical device render the proposed WLRS biosensor ideal for future point-of-need determination of carbendazim in food and environmental samples.

Author Contributions: conceptualization, G.K., C.-E.K., S.K. and E.L.; methodology, G.K., C.-E.K.; investigation, G.K., C.-E.K.; data curation, G.K., C.-E.K.; writing-original draft preparation, G.K., C.-E.K.; writing-review and editing, S.K., E.L.

Funding: This research is co-financed by Greece and the European Union (European Social Fund- ESF) through the Operational Programme «Human Resources Development, Education and Lifelong Learning 2014-2020» in the context of the project "Development of immunochemical analytical methodology, especially an ELISA and a biosensor assay, for determining benzimidazole-type pesticides" (MIS 5047811).

Acknowledgments: The authors would like to thank ThetaMetrisis SA for the kind supply of the WLRS instrumentation. They also thank the Animal House of NCSR "Demokritos" for excellent technical assistance in the development of the anti-carbendazim antibody (License No. 247871).

Conflicts of Interest: The authors declare no conflict of interest. The funders had no role in the design of the study; in the collection, analyses, or interpretation of data; in the writing of the manuscript, or in the decision to publish the results.

\section{References}

1. Karlsson, I.; Friberg, H.; Steinberg, C.; Persson, P. Fungicide effects on fungal community composition in the wheat phyllosphere. PLoS ONE 2014, 9, e111786. doi:10.1371/journal.pone.0111786.

2. Singh, S.; Singh, N.; Kumar, V.; Datta, S.; Wani, A.; Singh, D.; Singh, K.; Singh, J. Toxicity, monitoring and biodegradation of the fungicide carbendazim. Environ. Chem. Lett. 2016, 14, 317-329. doi:10.1007/s10311016-0566-2.

3. Tortella, G.R.; Mella-Herrera, R.A.; Sousa, D.Z.; Rubilar, O.; Briceño, G.; Parra, L.; Diez, M.C. Carbendazim dissipation in the biomixture of on-farm biopurification systems and its effect on microbial communities. Chemosphere 2013, 93, 1084-1093. doi:10.1016/j.chemosphere.2013.05.084.

4. Goodson, W.H.; Lowe, L.; Carpenter, D.O.; Gilbertson, M.; Ali, A.; Salsamendi, A.; Lasfar, A.; Carnero, A.; Azqueta, A.; Amedei, A.; et al. Assessing the carcinogenic potential of low-dose exposures to chemical mixtures in the environment: The challenge ahead. Carcinogenesis 2015, 36, 254-296. doi:10.1093/carcin/bgv039.

5. EU Pesticide Database. European Commission. 2015. Available online: http://ec. europa.eu/food/plant/pesticides/eu-pesticides database/public/?event=homepageandlanguage=EN (accessed on).

6. Hiemstra, M.; de Kok, A. Comprehensive multi-residue method for the target analysis of pesticides in crops using liquid chromatography-tandem mass spectrometry. J. Chromatogr. A 2007, 1154, 3-25. doi:10.1016/j.chroma.2007.03.123.

7. Chayata, H.; Lassalle, Y.; Nicol, É.; Manolikakes, S.; Souissi, Y.; Bourcier, S.; Gosmini, C.; Bouchonnet, S. Characterization of the ultraviolet-visible photoproducts of thiophanate-methyl using high performance liquid chromatography coupled with high resolution tandem mass spectrometry-Detection in grapes and tomatoes. J. Chromatogr. A 2016, 1441, 75-82. doi:10.1016/j.chroma.2016.02.078.

8. Gough, K.C.; Jarvis, S.; Maddison, B.C. Development of competitive immunoassays to hydroxyl containing fungicide metabolites. J. Environ. Sci. Health B 2011, 46, 581-589. doi:10.1080/03601234.2011.586596.

9. Guo, L.; Wu, X.; Liu, L.; Kuang, H.; Xu, C. Gold Nanoparticle-Based Paper Sensor for Simultaneous Detection of 11 Benzimidazoles by One Monoclonal Antibody. Small 2017, doi:10.1002/smll.201701782.

10. Reynoso, E.C.; Torres, E.; Bettazzi, F.; Palchetti, I. Trends and Perspectives in Immunosensors for Determination of Currently-Used Pesticides: The Case of Glyphosate, Organophosphates, and Neonicotinoids. Biosensors (Basel) 2019, 9, 20. doi:10.3390/bios9010020.

11. Zikos, C.; Evangelou, A.; Karachaliou, C.-E.; Gourma, G.; Blouchos, P.; Moschopoulou, G.; Yialouris, C.; Griffiths, J.; Johnson, G.; Petrou, P.; et al. Commercially available chemicals as immunizing haptens for the development of a polyclonal antibody recognizing carbendazim and other benzimidazole-type fungicides. Chemosphere 2015, 119, S16-S20. doi:10.1016/j.chemosphere.2014.03.049. 
Publisher's note: MDPI stays neutral with regard to jurisdictional claims in published maps and institutional affiliations.

(C) 2020 by the authors. Submitted for possible open access publication under the terms and conditions of the Creative Commons Attribution (CC BY) license (http://creativecommons.org/licenses/by/4.0/). 